\title{
EVALUATION OF BLACK GLUTINOUS RICE GENOTYPES FOR STABILITY OF GAMMA ORYZANOL AND YIELD IN TROPICAL ENVIRONMENTS
}

\author{
Poramate BANTERNG ${ }^{1,2^{*}}$ and Anchalee JORALEE \\ ${ }^{1}$ Khon Kaen University, Faculty of Agriculture, Department of Plant Science and \\ Agricultural Resources, Khon Kaen, THAILAND \\ ${ }^{2}$ Khon Kaen University, Faculty of Agriculture, Plant Breeding Research Center for \\ Sustainable Agriculture, Khon Kaen, THAILAND \\ *Corresponding author: bporam@kku.ac.th
}

Received: 29.09.2014

\begin{abstract}
Gamma oryzanol is an important phytochemical of black glutinous rice as it has positive effects on human health. The objective of this study was to evaluate stability of gamma oryzanol content and grain yield for black glutinous rice genotypes grown under different environments. Twenty-five black glutinous rice genotypes and 2 white glutinous rice genotypes were evaluated during 2011 and 2012 in 8 farmers' fields of Thailand. The experimental trials were laid out in a randomized complete blocks design (RCBD) with 3 replications. Soil data before growing, weather data during growing season, yield components, grain yield and gamma oryzanol content were recorded. The results indicated that location $(L)$, genotype $(G)$ and $G \times L$ interaction were highly significant for number of panicles per hill, number of filled grains per panicle, 1000-grain weight, grain yield, gamma oryzanol per grain weight and gamma oryzanol yield. The GGE biplot analysis indicated that KKU-GLBL-06-023 (V17), Khaokam Gs.no. 88084 (V5) and KKU-GL-BL-11-001 (V25) had both high mean performance and stability for grain yield. The superior genotypes for gamma oryzanol per grain weight were KKU-GL-BL11-001 (V25) and KKU-GL-BL-06-043 (V23). The desirable genotypes for gamma oryzanol yield were KKUGL-BL-11-001 (V25), Niewdam Gs.no. 21427 (V3) and KKU-GL-BL-06-043 (V23).
\end{abstract}

Key words: Breeding, Combined analysis, Germplasm, GGE biplot, Sticky rice

\section{INTRODUCTION}

Glutinous rice with purple or red color in pericarp (black glutinous rice) has been recognized in many countries of Asia. It has been used for making diverse products including functional food, drink, medicine and cosmetics. According to the popularity of healthy consumption trend in this present, black glutinous rice is considered as one of the crops with high potential for making such products. In addition, black glutinous rice is a crop thought to have herbal properties and it has been used traditionally in various kinds of medical treatments. The purple pigment (anthocyanin: cyaniding-3-glucoside) in the husk (hull) and pericarp and gamma oryzanol from rice bran oil are advantageous antioxidants (Xu et al., 2001; Juliano et al., 2005; Vorarat et al., 2010; Pitija et al., 2013). Black rice contained two to three times the amount of anthocyanin and gamma oryzanol higher than did white rice (Ryu et al., 1998; Boonsit et al., 2010). For gamma oryzanol, it is a mixture of phytosteryl ferulate esters in the rice bran oil (Bergman and Xu, 2003; Boonsit et al., 2010) with properties that are beneficial to health including improving the plasma lipid profile, reducing total plasma cholesterol, increasing high-density lipoprotein (HDL) cholesterol levels and inhibiting platelet aggregation (Cicero and Gaddi, 2001).

In Thailand, black glutinous rice has been increasingly attractive as a high value-added crop and it has become a key driving force in economic development. It is grown under rainfed condition in both upland and lowland environments. The farmers mostly grow local genotypes of black glutinous rice with low yield (Saenjum et al., 2012; Somsana et al., 2013). In order to improve the productivity, growing the suitable genotypes is an alternative option for sustainable farming systems. Therefore, growing the recommended rice genotypes with good adaptation to various environments and high yield is very important for the farmers. However, inconsistency of crop performance and yield in different environments has always been a problem and it also causes a big task for plant breeders (Romagosa and Fox, 1993; Yan and Hunt 2003; Sayar et al., 2013). The relative performances of rice genotypes for quantitative traits such as yield and gamma oryzanol content varied from one environment to another (Bergman and $\mathrm{Xu}, 2003$ ). If there is multitude of environment, it 
would also be difficult to develop suitable black glutinous rice genotypes for each environment. Thus, it seems more practical to determine and release a genotype with adaptation to more than a single environment. However, the research on this aspect for black glutinous rice in the tropical environments has been done very less so far. The objective of this study was to evaluate stability of gamma oryzanol content and grain yield for black glutinous rice genotypes grown under different environments.

\section{MATERIALS AND METHODS}

Twenty seven rice genotypes were used in this study (Table 1). Twenty-five black glutinous rice genotypes were obtained from Plant Breeding Research Center for Sustainable Agriculture, Faculty of Agriculture, Khon Kaen University, Thailand and 2 white glutinous rice genotypes were kindly donated from Rice Department of Thailand. The performances of all genotypes were evaluated in both upland and lowland conditions at a total of 8 farmers' fields during the main growing season of 2011 and 2012 in Khon Kaen province, Thailand (Table 2). The tested rice genotypes were laid out in a randomized complete blocks design (RCBD) with 3 replications. The entries were planted in the plots with $15 \mathrm{~m}^{2}$ in size. For upland condition, five seeds were planted per hill with a plant spacing of $30 \times 25 \mathrm{~cm}$ (Table 2) and seedlings were thinned to 3 plants per hill at 20 days after planting. All plots were fertilized at 45 days after planting with $\mathrm{N}, \mathrm{P}_{2} \mathrm{O}_{5}$ and $\mathrm{K}_{2} \mathrm{O}$ at the rate of $23.4 \mathrm{~kg} \mathrm{ha}^{-1}$. Urea fertilizer was also applied to all plots at the rate of $31.3 \mathrm{~kg} \mathrm{ha}^{-1}$ during early booting stage. For lowland condition, rice seedlings at approximate 30 days old after planting were transplanted with a plant spacing of $25 \times 25 \mathrm{~cm}$ and with 1 seedling per hill (Table 2). $\mathrm{N}, \mathrm{P}_{2} \mathrm{O}_{5}$ and $\mathrm{K}_{2} \mathrm{O}$ were applied for all experimental plots at 15 days after transplanting at the rate of 30,30 and $15 \mathrm{~kg} \mathrm{ha}^{-1}$, respectively. Urea fertilizer was applied at the rate of $31.3 \mathrm{~kg} \mathrm{ha}^{-1}$ during early booting stage. For both upland and lowland conditions, supplemental irrigation was supplied during the dry periods in the rainy season. Weeds, pests and diseases were controlled as necessary.

Table 1. Glutinous rice genotypes used for the study.

\begin{tabular}{clcl}
\hline No. & Genotype name & No. & Genotype name \\
\hline 1 & Niewdam Gs.no. 00621 (V1) & 15 & KKU-GL-BL-05-010 (V15) \\
2 & Niewdam Gs.no. 09475(V2) & 16 & KKU-GL-BL-05-011 (V16) \\
3 & Niewdam Gs.no. 21427 (V3) & 17 & KKU-GL-BL-06-023 (V17) \\
4 & Niewdam Gs.no. 21629 (V4) & 18 & KKU-GL-BL-06-034 (V18) \\
5 & Khaokam Gs.no. 88084(V5) & 19 & KKU-GL-BL-06-035 (V19) \\
6 & Khaokam Gs.no. 87090(V6) & 20 & KKU-GL-BL-06-038 (V20) \\
7 & KKU-GL-BL-05-001 (V7) & 21 & KKU-GL-BL-06-039 (V21) \\
8 & KKU-GL-BL-05-002(V8) & 22 & KKU-GL-BL-06-041 (V22) \\
9 & KKU-GL-BL-05-003 (V9) & 23 & KKU-GL-BL-06-043 (V23) \\
10 & KKU-GL-BL-05-004 (V10) & 24 & KKU-GL-BL-06-050 (V24) \\
11 & KKU-GL-BL-05-005 (V11) & 25 & KKU-GL-BL-11-001 (V25) \\
12 & KKU-GL-BL-05-006 (V12) & 26 & RD6 (V26) \\
13 & KKU-GL-BL-05-008 (V13) & 27 & HY 71 (V27) \\
14 & KKU-GL-BL-05-009 (V14) & & \\
\hline
\end{tabular}

Table 2. Location, land type and crop management for each experiment in Khon Kaen province, Thailand.

\begin{tabular}{llll}
\hline Location & Land type & Planting/Transplanting date & Spacing (cm x cm) \\
\hline 1. Ban Kham Pom (L1) & Upland & 21 June 2011 & $30 \times 25$ \\
2. Ban Wang Wa (L2) & Upland & 18 June 2011 & $30 \times 25$ \\
3. Ban Koh Nang Ngam (L3) & Lowland & 6 August 2011 & $25 \times 25$ \\
4. Ban Muang (L4) & Lowland & 8 August 2011 & $25 \times 25$ \\
5. Ban Kham Pom (L5) & Upland & 23 June 2012 & $30 \times 25$ \\
6. Ban Wang Wa (L6) & Upland & 19 June 2012 & $30 \times 25$ \\
7. Ban Nong Por (L7) & Lowland & 14 August 2012 & $25 \times 25$ \\
8. Ban Muang (L8) & Lowland & 9 August 2012 & $25 \times 25$ \\
\hline
\end{tabular}

For data collection, soil samples were collected from the experimental sites before the experiment was conducted at 3 spots and at the depths of $0-15 \mathrm{~cm}$. The soil samples were then analysed for physical and chemical properties. Maximum and minimum air temperatures and rainfall during growing period were recorded from the nearest meteorological stations (Table 3). At final harvest, the 10 sub-samples of each experimental plot were randomly chosen to determine number of panicles per hill, number of filled grains per panicle and 1,000-grain weight. The center rows in each experimental plot were harvested to observe grain yield. In addition, gamma oryzanol values from rice bran of 27 tested genotypes were also recorded by using high performance liquid chomatography (HPLC) method (Xu and Godber, 1999). Analysis of variance for individual locations and combined analysis of variances of 8 locations were performed by following the procedure described by Gomez and Gomez (1984). Duncan's multiple range test 
(DMRT) was also used to compare mean differences. All calculations were done using MSTAT-C package (Freed et al., 1989). Stability analysis was done by the GGE biplot method using the GGE biplot software (Yan, 2001). The
GGE biplot was constructed from the first two components (PC1 and PC2) that were derived from subjecting environment-centered crop data to singular value decomposition (SVD).

Table 3. Soil property before land preparation and weather condition during the growing season for each experiment.

\begin{tabular}{|c|c|c|c|c|c|c|c|c|c|}
\hline \multirow[b]{2}{*}{ Location } & \multicolumn{6}{|c|}{ Soil property } & \multicolumn{3}{|c|}{ Weather condition } \\
\hline & $\begin{array}{l}\text { Textural } \\
\text { class }\end{array}$ & $\begin{array}{l}\text { Total } \\
\mathbf{N}(\%)\end{array}$ & $\begin{array}{l}\text { Available } \\
\mathbf{P}(\text { ppm })\end{array}$ & $\begin{array}{l}\text { Exchangeable } \\
\text { K (ppm) }\end{array}$ & $\begin{array}{l}\text { C.E.C. } \\
(\text { c mol kg-1) }\end{array}$ & pH & $\begin{array}{l}\text { Maximum } \\
\text { temperature } \\
\left({ }^{\circ} \mathrm{C}\right)\end{array}$ & $\begin{array}{l}\text { Minimum } \\
\text { temperature } \\
\left({ }^{\circ} \mathrm{C}\right)\end{array}$ & $\begin{array}{l}\text { Rainfall } \\
(\mathbf{m m})\end{array}$ \\
\hline 1. Ban Kham Pom (L1) & Sand & 0.02 & 15.5 & 31.6 & 1.7 & 5.5 & 31.60 & 23.12 & 864.4 \\
\hline 2. Ban Wang Wa (L2) & Sand & 0.01 & 8.2 & 19.3 & 3.9 & 5.3 & 31.65 & 23.21 & 865.9 \\
\hline 3. Ban Koh Nang Ngam (L3) & $\begin{array}{l}\text { Sandy } \\
\text { Loam }\end{array}$ & 0.10 & 9.2 & 100.8 & 14.1 & 5.2 & 31.53 & 23.17 & 1064.1 \\
\hline 4. Ban Muang (L4) & Sand & 0.03 & 5.4 & 47.5 & 3.9 & 5.4 & 31.53 & 23.21 & 1064.1 \\
\hline 5. Ban Kham Pom (L5) & Sand & 0.01 & 10.9 & 34.9 & 2.9 & 5.1 & 32.79 & 23.41 & 528.2 \\
\hline 6. Ban Wang Wa (L6) & Sand & 0.02 & 8.8 & 53.3 & 0.3 & 5.1 & 32.78 & 23.44 & 528.2 \\
\hline 7. Ban Nong Por (L7) & $\begin{array}{l}\text { Sandy } \\
\text { Loam }\end{array}$ & 0.02 & 3.6 & 52.4 & 2.8 & 6.2 & 32.53 & 23.66 & 544.4 \\
\hline 8. Ban Muang (L8) & Sand & 0.02 & 6.4 & 35.9 & 1.6 & 4.8 & 32.54 & 23.67 & 544.4 \\
\hline
\end{tabular}

\section{RESULTS AND DICUSSION}

\section{Combined analysis of variance}

Location (L), genotype $(\mathrm{G})$ and $\mathrm{G} \times \mathrm{L}$ interaction were highly significant $(p<0.01)$ for number of panicles per hill, number of filled grains per panicle, 1000-grain weight, grain yield, gamma oryzanol per grain weight and gamma oryzanol yield (Table 4$)$. The variations due to locations shared the largest portions of total variations for number of panicles per hill (45.7\%), number of filled grains per panicle $(52.2 \%)$ and 1000 -grain weight $(70.7 \%)$. The $\mathrm{G} \times \mathrm{L}$ interactions contributed to the large portions of total variations for grain yield $(36.0 \%)$, gamma oryzanol per grain weight $(32.7 \%)$ and gamma oryzanol yield (35.6\%). The contributions of genotypic variations were rather small for number of panicles per hill, number of filled grains per panicle, 1000-grain weight, grain yield and gamma oryzanol yield when compared to the variation of locations. This indicated that growing environments had greater effect on these crop performances than did genotypes. In order to select the genotypes with optimized levels of these crop characteristics, therefore, it is necessary to grow the breeding materials in multiple years and environments.

Table 4. Mean squares for number of panicles per hill, number of filled grains per panicle, 1000-grain weight (g), grain yield $\left(\mathrm{kg} \mathrm{ha}^{-1}\right)$, gamma oryzanol per grain weight ( $\mathrm{mg} \mathrm{g}^{-1}$ grain) and gamma oryzanol yield $\left(\mathrm{g} \mathrm{ha}^{-1}\right)$ for 27 tested genotypes in 8 locations.

\begin{tabular}{|c|c|c|c|c|c|c|c|}
\hline \multirow[b]{2}{*}{$\begin{array}{l}\text { Source of } \\
\text { variation }\end{array}$} & \multirow[b]{2}{*}{$\begin{array}{l}\text { Degree } \\
\text { of } \\
\text { freedom }\end{array}$} & \multicolumn{6}{|c|}{ Mean square } \\
\hline & & $\begin{array}{l}\text { Number of } \\
\text { panicles per } \\
\text { hill }\end{array}$ & $\begin{array}{l}\text { Number of filled } \\
\text { grains per } \\
\text { panicle }\end{array}$ & $\begin{array}{l}\text { 1000-grain } \\
\text { weight (g) }\end{array}$ & $\begin{array}{l}\text { Grain yield } \\
\left(\mathrm{kg} \mathrm{ha}^{-1}\right)\end{array}$ & $\begin{array}{l}\text { Gamma oryzanol } \\
\text { per grain weight } \\
\text { (mg g grain) }\end{array}$ & $\begin{array}{l}\text { Gamma oryzanol } \\
\text { yield } \\
\left(\mathrm{g} \mathrm{ha}^{-1}\right)\end{array}$ \\
\hline Location (L) & 7 & $150.1(45.7)^{* *}$ & $30230.4(52.2)^{* *}$ & $839.6(70.7)^{* * *}$ & $8632369.0(27.6)^{* * *}$ & $0.08909(28.3)^{* *}$ & $961561.0(29.4)^{* *}$ \\
\hline Replication/L & 16 & $6.4(4.5)$ & $547.8(2.2)$ & $3.7(0.7)$ & $312980.0(2.3)$ & $0.00223(1.6)$ & $61636.0(4.3)$ \\
\hline Genotype (G) & 26 & $11.8(13.4)^{* *}$ & $2778.7(17.8)^{* *}$ & $30.5(9.5)^{* *}$ & $1036843.0(12.3)^{* *}$ & $0.0254(29.9)^{* *}$ & $86254.0(9.8)^{* *}$ \\
\hline $\mathrm{G} \times \mathrm{L}$ & 182 & $3.0(23.8)^{* *}$ & $291.3(13.1)^{* *}$ & $5.7(12.5)^{* *}$ & $432147.0(36.0)^{* *}$ & $0.00396(32.7)^{* *}$ & $44827.0(35.6)^{* *}$ \\
\hline Pooled error & 416 & $0.7(12.7)$ & $143.9(14.8)$ & $1.3(6.5)$ & $114207.0(21.7)$ & $0.0004(7.5)$ & $11504.0(20.9)$ \\
\hline Total & 647 & & & & & & \\
\hline \%C.V. (GxE) & & 33.39 & 23.68 & 7.57 & 31.8 & 17.98 & 54.38 \\
\hline \%C.V. (GxRxE) & & 11.16 & 12.14 & 4.43 & 19.21 & 7.64 & 23.5 \\
\hline
\end{tabular}

Numbers within the parentheses are percent (\%) of sum squares to total sum of squares.

$* *$ significant difference $(p<0.01)$

For gamma oryzanol per grain weight, the results showed that genotypic variation was slightly higher than the variation due to locations, whereas the $\mathrm{G} \times \mathrm{L}$ interaction was observed. Therefore, the experiments in various environments are also still important for selecting rice genotypes with high level of this trait. A report from Bergman and $\mathrm{Xu}$ (2003) indicated that genotype and environment played a significant role in controlling gamma oryzanol per grain weight. They also suggested evaluation of the tested genotypes in multiple environments before selecting the best genotype with enhanced gamma oryzanol level.

\section{Range of the tested environments}

There were significant differences $(p<0.01)$ for all crop traits among 8 environments. This could be explained by the differences in the weather and soil conditions as well as management practices. Means for all tested environments ranged from 5.90-9.27 for number of panicles per hill, 77.00-130.60 for number of filled grains per panicle, 21.79 $29.26 \mathrm{~g}$ for 1000 -grain weight, $1274.50-2155.27 \mathrm{~kg} \mathrm{ha}^{-1}$ for grain yield, $0.224-0.323 \mathrm{mg} \mathrm{g}^{-1}$ grain for gamma oryzanol per grain weight and 323.83-662.64 $\mathrm{g} \mathrm{ha}^{-1}$ for gamma oryzanol yield (Table 5). 
Ban Koh Nang Ngam produced the highest values for number of filled grains per panicle, 1000-grain weight, grain yield, gamma oryzanol per grain weight and gamma oryzanol yield because Ban Koh Nang Ngam is a lowland environment with higher soil fertility as well as high total amount of rain fall during the crop duration (Table 2 and 3 ). In order to select the suitable location for yield and gamma oryzanol production, therefore, this location would be identified as more favorable environment for crop growth when compared with the others tested locations.

Table 5. Mean performance for number of panicles per hill, number of filled grains per panicle, 1000-grain weight (g), yield $\left(\mathrm{kg} \mathrm{ha}^{-1}\right)$, gamma oryzanol per grain weight $\left(\mathrm{mg} \mathrm{g}^{-1}\right.$ grain) and gamma oryzanol yield $\left(\mathrm{kg} \mathrm{ha}^{-1}\right)$ of 8 locations.

\begin{tabular}{|c|c|c|c|c|c|c|}
\hline Location & $\begin{array}{l}\text { Number of } \\
\text { panicles } \\
\text { per hill }\end{array}$ & $\begin{array}{l}\text { Number } \\
\text { of filled } \\
\text { grains per } \\
\text { panicle }\end{array}$ & $\begin{array}{l}\text { 1000- } \\
\text { grain } \\
\text { weight (g) }\end{array}$ & $\begin{array}{l}\text { Grain yield } \\
\left(\mathrm{kg} \mathrm{ha}^{-1}\right)\end{array}$ & $\begin{array}{l}\text { Gamma } \\
\text { oryzanol per } \\
\text { grain weight } \\
\left(\mathrm{mg} \mathrm{g}^{-1} \text { grain) }\right.\end{array}$ & $\begin{array}{l}\text { Gamma } \\
\text { oryzanol yield } \\
\left(\mathrm{g} \mathrm{ha}^{-1}\right)\end{array}$ \\
\hline 1. Ban Kham Pom (L1) & $8.49 \mathrm{~b}$ & $113.11 \mathrm{~b}$ & $27.29 \mathrm{c}$ & $2155.27 \mathrm{a}$ & $0.224 \mathrm{~g}$ & $476.50 \mathrm{~b}$ \\
\hline 2. Ban Wang Wa (L2) & $8.08 \mathrm{c}$ & $98.01 \mathrm{c}$ & $28.61 \mathrm{~b}$ & $1909.62 \mathrm{~b}$ & $0.269 \mathrm{c}$ & $502.93 \mathrm{~b}$ \\
\hline 3. Ban Koh Nang Ngam (L3) & $6.18 \mathrm{~d}$ & $130.60 \mathrm{a}$ & $29.26 \mathrm{a}$ & $2097.35 \mathrm{a}$ & $0.323 \mathrm{a}$ & $662.64 \mathrm{a}$ \\
\hline 4. Ban Muang (L4) & $5.95 \mathrm{~d}$ & $112.18 \mathrm{~b}$ & $28.68 \mathrm{~b}$ & $1689.14 \mathrm{c}$ & $0.298 \mathrm{~b}$ & $483.19 \mathrm{~b}$ \\
\hline 5. Ban Kham Pom (L5) & $8.34 \mathrm{bc}$ & $81.97 \mathrm{~d}$ & $21.79 \mathrm{f}$ & $1331.90 \mathrm{~d}$ & $0.236 \mathrm{f}$ & $323.83 \mathrm{~d}$ \\
\hline 6. Ban Wang Wa (L6) & $9.27 \mathrm{a}$ & $77.45 \mathrm{~d}$ & $23.78 \mathrm{~d}$ & $1696.30 \mathrm{c}$ & 0.243 ef & $400.77 \mathrm{c}$ \\
\hline 7. Ban Nong Por (L7) & $8.57 \mathrm{~b}$ & $100.24 \mathrm{c}$ & $23.04 \mathrm{e}$ & $1919.80 \mathrm{~b}$ & $0.250 \mathrm{de}$ & $472.83 \mathrm{~b}$ \\
\hline 8. Ban Muang (L8) & $5.90 \mathrm{~d}$ & $77.00 \mathrm{~d}$ & $21.92 \mathrm{f}$ & $1274.50 \mathrm{~d}$ & $0.257 \mathrm{~d}$ & $328.56 \mathrm{~d}$ \\
\hline
\end{tabular}

Values in the column followed by the same letter (s) are not significantly different at the $p<0.01$ level by DMRT.

\section{Performance and stability of rice genotypes}

The results indicated significant differences $(p<0.01)$ among tested genotypes for all crop traits. KKU-GL-BL05-005 (V11) and KKU-GL-BL-05-008 (V13) had the highest means for number of panicles per hill with 8.66 and 8.64 panicles per hill, respectively. Whereas the highest means for number of filled grains per panicle were found for Niewdam Gs.no. 21427 (V3) (120.10) and Niewdam Gs.no. 00621 (V1) (116.73) (Table 6). For 1000-grain weight, KKU-GL-BL-05-004 (V10) and KKU-GL-BL-05001 (V7) gave the highest values with 27.36 and $27.34 \mathrm{~g}$, respectively.

RD6 (V26) gave the highest value of grain yield (2304 $\mathrm{kg} \mathrm{ha}^{-1}$ ) but the values for gamma oryzanol per grain weight (0.168 $\mathrm{mg} \mathrm{g}^{-1}$ grain) and gamma oryzanol yield (409.4 $\mathrm{g} \mathrm{ha}^{-}$ $\left.{ }^{1}\right)$ were rather low when compared with the other genotypes (Table 7). Niewdam Gs.no. 21427 (V3) and KKU-GL-BL11-001 (V25) had the highest means for gamma oryzanol per grain weight with 0.327 and $0.318 \mathrm{mg} \mathrm{g}^{-1}$ grain, respectively, whereas the highest mean for gamma oryzanol yield was found for Niewdam Gs.no. 21629 (V4) $\left(564.4 \mathrm{~g} \mathrm{ha}^{-1}\right)$. Therefore, these genotypes with high mean performance would be recommended as the parental sources for further development of new rice genotypes with high productivity for both grain yield and gamma oryzanol content in plant breeding programs.

Based on gamma oryzanol per grain weight, the results also indicated that all 25 black glutinous rice genotypes had higher amount of gamma oryzanol per grain weight than did 2 white rice check genotypes, suggesting that these particular black rice genotypes would also have a higher amount of health and medicinal substances. Furthermore, anthocyanin involving free radical scavenging and antioxidant is also the main phytochemical in black rice (Pitija et al., 2013). Thus, black rice would be more advantageous for medicinal property and it is also a rice genetic resource for improving rice as a functional food crop. 
Table 6. Mean performance for number of panicles per hill, number of filled grains per panicle and 1000-grain weight (g) of 27 tested genotypes in 8 locations.

\begin{tabular}{lccc}
\hline Genotype name & Number of panicles per hill & $\begin{array}{c}\text { Number of filled grains per } \\
\text { panicle }\end{array}$ & 1000-grain weight (g) \\
\hline Niewdam Gs.no. 00621 (V1) & $5.75 \mathrm{i}$ & $116.73 \mathrm{a}$ & $26.21 \mathrm{c}-\mathrm{f}$ \\
Niewdam Gs.no. 09475 (V2) & $7.09 \mathrm{efg}$ & $106.55 \mathrm{bc}$ & $24.65 \mathrm{~h}-\mathrm{k}$ \\
Niewdam Gs.no. 21427 (V3) & $6.38 \mathrm{~h}$ & $120.10 \mathrm{a}$ & $25.24 \mathrm{~g}-\mathrm{j}$ \\
Niewdam Gs.no. 21629 (V4) & $8.17 \mathrm{a}-\mathrm{d}$ & $100.96 \mathrm{c}-\mathrm{g}$ & $23.90 \mathrm{k}$ \\
Khaokam Gs.no. 88084 (V5) & $8.32 \mathrm{abc}$ & $94.36 \mathrm{~d}-\mathrm{g}$ & $24.13 \mathrm{k}$ \\
Khaokam Gs.no. 87090 (V6) & $7.53 \mathrm{def}$ & $104.53 \mathrm{bcd}$ & $24.58 \mathrm{~h}-\mathrm{k}$ \\
KKU-GL-BL-05-001 (V7) & $7.74 \mathrm{cde}$ & $92.05 \mathrm{gh}$ & $27.34 \mathrm{a}$ \\
KKU-GL-BL-05-002 (V8) & $7.44 \mathrm{efg}$ & $94.63 \mathrm{~d}-\mathrm{g}$ & $23.96 \mathrm{k}$ \\
KKU-GL-BL-05-003 (V9) & $8.37 \mathrm{abc}$ & $83.79 \mathrm{hi}$ & $26.85 \mathrm{a}-\mathrm{d}$ \\
KKU-GL-BL-05-004 (V10) & $8.49 \mathrm{ab}$ & $77.54 \mathrm{i}$ & $27.36 \mathrm{a}$ \\
KKU-GL-BL-05-005 (V11) & $8.66 \mathrm{a}$ & $27.10 \mathrm{abc}$ \\
KKU-GL-BL-05-006 (V12) & $6.75 \mathrm{gh}$ & $76.41 \mathrm{i}$ & $24.66 \mathrm{~h}-\mathrm{k}$ \\
KKU-GL-BL-05-008 (V13) & $8.64 \mathrm{a}$ & $107.2 \mathrm{bc}$ & $26.13 \mathrm{~d}-\mathrm{g}$ \\
KKU-GL-BL-05-009 (V14) & $7.78 \mathrm{~b}-\mathrm{e}$ & $93.59 \mathrm{e}-\mathrm{h}$ & $25.86 \mathrm{efg}$ \\
KKU-GL-BL-05-010 (V15) & $7.75 \mathrm{cde}$ & $92.63 \mathrm{fgh}$ & $24.19 \mathrm{k}$ \\
KKU-GL-BL-05-011 (V16) & $7.45 \mathrm{~d}-\mathrm{g}$ & $83.63 \mathrm{hi}$ & $26.43 \mathrm{a}-\mathrm{f}$ \\
KKU-GL-BL-06-023 (V17) & $8.36 \mathrm{abc}$ & $113.83 \mathrm{ab}$ & $27.26 \mathrm{ab}$ \\
KKU-GL-BL-06-034 (V18) & $7.27 \mathrm{efg}$ & $93.44 \mathrm{e}-\mathrm{h}$ & $25.20 \mathrm{~g}-\mathrm{j}$ \\
KKU-GL-BL-06-035 (V19) & $6.90 \mathrm{fgh}$ & $107.06 \mathrm{bc}$ & $26.38 \mathrm{~b}-\mathrm{f}$ \\
KKU-GL-BL-06-038 (V20) & $7.20 \mathrm{efg}$ & $103.91 \mathrm{~b}-\mathrm{e}$ & $25.55 \mathrm{fgh}$ \\
KKU-GL-BL-06-039 (V21) & $7.71 \mathrm{cde}$ & $98.13 \mathrm{c}-\mathrm{g}$ & $25.49 \mathrm{f}-\mathrm{i}$ \\
KKU-GL-BL-06-041 (V22) & $7.24 \mathrm{efg}$ & $98.09 \mathrm{c}-\mathrm{g}$ & $26.23 \mathrm{c}-\mathrm{f}$ \\
KKU-GL-BL-06-043 (V23) & $7.37 \mathrm{efg}$ & $103.44 \mathrm{cde}$ & $24.75 \mathrm{~h}-\mathrm{k}$ \\
KKU-GL-BL-06-050 (V24) & $7.53 \mathrm{def}$ & $100.54 \mathrm{c}-\mathrm{g}$ & $26.66 \mathrm{a}-\mathrm{e}$ \\
KKU-GL-BL-11-001 (V25) & $7.18 \mathrm{efg}$ & $102.61 \mathrm{c}-\mathrm{f}$ & $24.54 \mathrm{ijk}$ \\
RD6 (V26) & $8.34 \mathrm{abc}$ & $103.6 \mathrm{cde}$ & $24.64 \mathrm{~h}-\mathrm{k}$ \\
HY 71 (V27) & $7.79 \mathrm{~b}-\mathrm{e}$ & $106.45 \mathrm{bc}$ & $24.49 \mathrm{jk}$ \\
\hline Ve & $92.46 \mathrm{fgh}$ & \\
\hline
\end{tabular}

Values in the column followed by the same letter (s) are not significantly different at the $p<0.01$ level by DMRT.

Table 7. Mean performance and ranking for yield $\left(\mathrm{kg} \mathrm{ha}^{-1}\right)$, gamma oryzanol per grain weight $\left(\mathrm{mg} \mathrm{g}^{-1}\right.$ grain) and gamma oryzanol yield $\left(\mathrm{kg} \mathrm{ha}^{-1}\right)$ of 27 tested genotypes in 8 locations.

\begin{tabular}{|c|c|c|c|c|c|c|}
\hline \multirow[t]{2}{*}{ Genotype name } & \multicolumn{2}{|l|}{ Grain yield } & \multicolumn{2}{|c|}{$\begin{array}{c}\text { Gamma oryzanol per grain } \\
\text { weight }\end{array}$} & \multicolumn{2}{|c|}{ Gamma oryzanol yield } \\
\hline & $\begin{array}{c}\text { Mean } \\
\left(\mathrm{kg} \mathrm{ha}^{-1}\right)\end{array}$ & Rank & $\begin{array}{c}\text { Mean } \\
\left(\mathrm{mg} \mathrm{g}^{-1} \text { grain }\right)\end{array}$ & Rank & $\begin{array}{c}\text { Mean } \\
\left(\mathrm{g} \mathrm{ha}^{-1}\right)\end{array}$ & Rank \\
\hline RD6 (V26) & $2304 \mathrm{a}$ & 1 & $0.168 \mathrm{n}$ & 27 & $409.4 \mathrm{f}-\mathrm{i}$ & 21 \\
\hline KKU-GL-BL-06-023 (V17) & $2203 \mathrm{ab}$ & 2 & $0.223 \mathrm{~m}$ & 25 & $487.0 \mathrm{a}-\mathrm{f}$ & 9 \\
\hline Khaokam Gs.no. 88084 (V5) & $2023 \mathrm{bc}$ & 3 & $0.247 \mathrm{kl}$ & 21 & 506.4 a-e & 6 \\
\hline KKU-GL-BL-05-001 (V7) & $2012 \mathrm{bc}$ & 4 & $0.245 \mathrm{kl}$ & 22 & $491.7 \mathrm{a}-\mathrm{f}$ & 8 \\
\hline Niewdam Gs.no. 21629 (V4) & $1998 \mathrm{bc}$ & 5 & 0.285 cde & 6 & $564.4 \mathrm{a}$ & 1 \\
\hline KKU-GL-BL-06-050 (V24) & $1872 \mathrm{~cd}$ & 6 & $0.262 \mathrm{~h}-\mathrm{k}$ & 15 & 480.1 a-f & 10 \\
\hline KKU-GL-BL-05-011 (V16) & 1810 cde & 7 & 0.2421 & 23 & $427.9 \mathrm{~d}-\mathrm{i}$ & 17 \\
\hline KKU-GL-BL-11-001 (V25) & 1801 cde & 8 & $0.318 \mathrm{a}$ & 2 & $556.7 \mathrm{ab}$ & 3 \\
\hline HY 71 (V27) & 1792 cde & 9 & $0.214 \mathrm{~m}$ & 26 & $400.8 \mathrm{f}-\mathrm{i}$ & 24 \\
\hline KKU-GL-BL-05-008 (V13) & 1789 cde & 10 & $0.252 \mathrm{i}-1$ & 18 & $453.1 \mathrm{c}-\mathrm{h}$ & 14 \\
\hline KKU-GL-BL-06-043 (V23) & 1788 cde & 11 & $0.302 \mathrm{~b}$ & 3 & $531.3 \mathrm{abc}$ & 4 \\
\hline Niewdam Gs.no. 09475 (V2) & 1782 cde & 12 & $0.289 \mathrm{bc}$ & 4 & $520.5 \mathrm{a}-\mathrm{d}$ & 5 \\
\hline KKU-GL-BL-06-041 (V22) & 1763 cde & 13 & $0.28 \mathrm{c}-\mathrm{g}$ & 9 & $492.1 \mathrm{a}-\mathrm{f}$ & 7 \\
\hline Niewdam Gs.no. 21427 (V3) & $1752 \mathrm{c}-\mathrm{f}$ & 14 & $0.327 \mathrm{a}^{\circ}$ & 1 & $561.7 \mathrm{ab}$ & 2 \\
\hline Khaokam Gs.no. 87090 (V6) & $1682 \mathrm{~d}-\mathrm{g}$ & 15 & 0.269 e-h & 11 & $447.8 \mathrm{c}-\mathrm{h}$ & 15 \\
\hline KKU-GL-BL-06-034 (V18) & $1673 \mathrm{~d}-\mathrm{g}$ & 16 & $0.262 \mathrm{~h}-\mathrm{k}$ & 14 & $407.6 \mathrm{f}-\mathrm{i}$ & 22 \\
\hline KKU-GL-BL-05-005 (V11) & $1661 \mathrm{~d}-\mathrm{g}$ & 17 & $0.272 \mathrm{~d}-\mathrm{h}$ & 10 & $442.2 \mathrm{c}-\mathrm{i}$ & 16 \\
\hline Niewdam Gs.no. 00621 (V1) & $1658 \mathrm{~d}-\mathrm{g}$ & 18 & $0.282 \mathrm{c}-\mathrm{f}$ & 8 & $477.1 \mathrm{a}-\mathrm{f}$ & 11 \\
\hline KKU-GL-BL-06-038 (V20) & $1644 \mathrm{~d}-\mathrm{g}$ & 19 & $0.288 \mathrm{bcd}$ & 5 & $470.0 \mathrm{~b}-\mathrm{g}$ & 12 \\
\hline KKU-GL-BL-05-003 (V9) & $1634 \mathrm{~d}-\mathrm{g}$ & 20 & $0.259 \mathrm{~h}-\mathrm{k}$ & 17 & $411.0 \mathrm{f}-\mathrm{i}^{\circ}$ & 20 \\
\hline KKU-GL-BL-05-004 (V10) & $1633 \mathrm{~d}-\mathrm{g}$ & 21 & $0.261 \mathrm{~h}-\mathrm{k}$ & 16 & $417.1 \mathrm{e}-\mathrm{i}$ & 18 \\
\hline KKU-GL-BL-05-006 (V12) & $1627 \mathrm{~d}-\mathrm{g}$ & 22 & $0.227 \mathrm{~m}$ & 24 & $378.9 \mathrm{ghi}$ & 25 \\
\hline KKU-GL-BL-05-009 (V14) & $1619 \mathrm{~d}-\mathrm{g}$ & 23 & 0.285 cde & 7 & $461.7 \mathrm{c}-\mathrm{g}$ & 13 \\
\hline KKU-GL-BL-06-035 (V19) & $1600 \mathrm{~d}-\mathrm{g}$ & 24 & $0.248 \mathrm{kl}$ & 20 & $401.4 \mathrm{f}-\mathrm{i}$ & 23 \\
\hline KKU-GL-BL-06-039 (V21) & 1534 efg & 25 & $0.267 \mathrm{f}-\mathrm{i}$ & 12 & $415.2 \mathrm{e}-\mathrm{i}$ & 19 \\
\hline KKU-GL-BL-05-002 (V8) & $1460 \mathrm{fg}$ & 26 & $0.249 \mathrm{jkl}$ & 19 & $360.1 \mathrm{hi}$ & 26 \\
\hline KKU-GL-BL-05-010 (V15) & $1387 \mathrm{~g}$ & 27 & $0.265 \mathrm{~g}-\mathrm{j}$ & 13 & $349.8 \mathrm{i}$ & 27 \\
\hline
\end{tabular}

Values in the column followed by the same letter (s) are not significantly different at the $p<0.01$ level by DMRT.

The significance of $\mathrm{G} \times \mathrm{L}$ interactions for all crop traits indicated the differential response of varying genotypes under change in the environments and the difficulty in identifying the superior genotypes. High $\mathrm{G} \times \mathrm{L}$ interaction complicates plant breeding programs to explore the superior genotypes in various environments (Comstock and Moll, 1963). Numerous methods have been commonly proposed to reveal patterns of interaction between 
genotypes and environments and to assess stability of the genotypes across various environments. In present, however, the GGE biplot method is an effective tool to consider both $\mathrm{G}$ and $\mathrm{G} \times \mathrm{L}$ interaction effects. It allows visual examination of the relationships among the test environments, genotypes and the $\mathrm{G} \times \mathrm{L}$ interactions (Yan, 2001). Therefore, it was used to evaluate mean performance and stability of each tested genotype in this study.

The results of mean performance and stability evaluation of 27 rice genotypes by GGE biplot method are shown in Figure 1 for number of panicles per hill, number of filled grains per panicle and 1,000-grain weight and Figure 2 for yield, gamma oryzanol per grain weight and gamma oryzanol yield. An ideal genotype should have high mean performance and be absolutely stable. In each figure, a small circle in the center of a biplot indicates average environment coordination (AEC) which is the average of environmental PC1 and PC2 scores. The center of the concentric circles represents the position of a reference for genotype evaluation. A genotype is more favorable if it is closer to the concentric circles. The genotype on the right side of the line with double arrows has yield performance greater than mean yield, whereas the genotype on the left side of this line has yield less than mean yield. The ideal position is defined by a projection on the meanenvironment axis that equals the longest vector of the genotypes having above-average mean yield and by a zero projection on to the perpendicular line (Yan and Hunt 2003).

Based on the results from GGE biplot, there were different desirable genotypes in different crop traits. KKUGL-BL-05-008 (V13), KKU-GL-BL-06-023 (V17), RD6 (V26) and Khaokam Gs.no. 88084 (V5) were close to the concentric center, and they can be considered as genotypes with both high mean performance and stability for number of panicles per hill (Figure 1), whereas the genotypes with high mean performance and stability for number of filled grains per panicle were KKU-GL-BL-05-006 (V12), Niewdam Gs.no. 09475 (V2) and RD6 (V26). For 1,000grain weight, KKU-GL-BL-05-004 (V10), KKU-GL-BL06-050 (V24), KKU-GL-BL-05-011 (V16) and KKU-GLBL-06-035 (V19) showed good performance in both mean and stability when compared to the other genotypes.

According to GGE biplot for grain yield in Figure 2, more close to concentric circle indicated that KKU-GL-BL06-023 (V17), Khaokam Gs.no. 88084 (V5) and KKU-GLBL-11-001 (V25) had both high mean performance and stability. RD6 (V26) had high mean performance genotype as it was located on far right hand side of the line with double arrows. However, a long projection on to the perpendicular line indicated that this genotype had rather low yield stability. The best genotypes in term of both high mean performance and stability for gamma oryzanol per grain weight were KKU-GL-BL-11-001 (V25) and KKUGL-BL-06-043 (V23), whereas the best genotypes for gamma oryzanol yield were KKU-GL-BL-11-001 (V25), Niewdam Gs.no. 21427 (V3) and KKU-GL-BL-06-043 (V23).
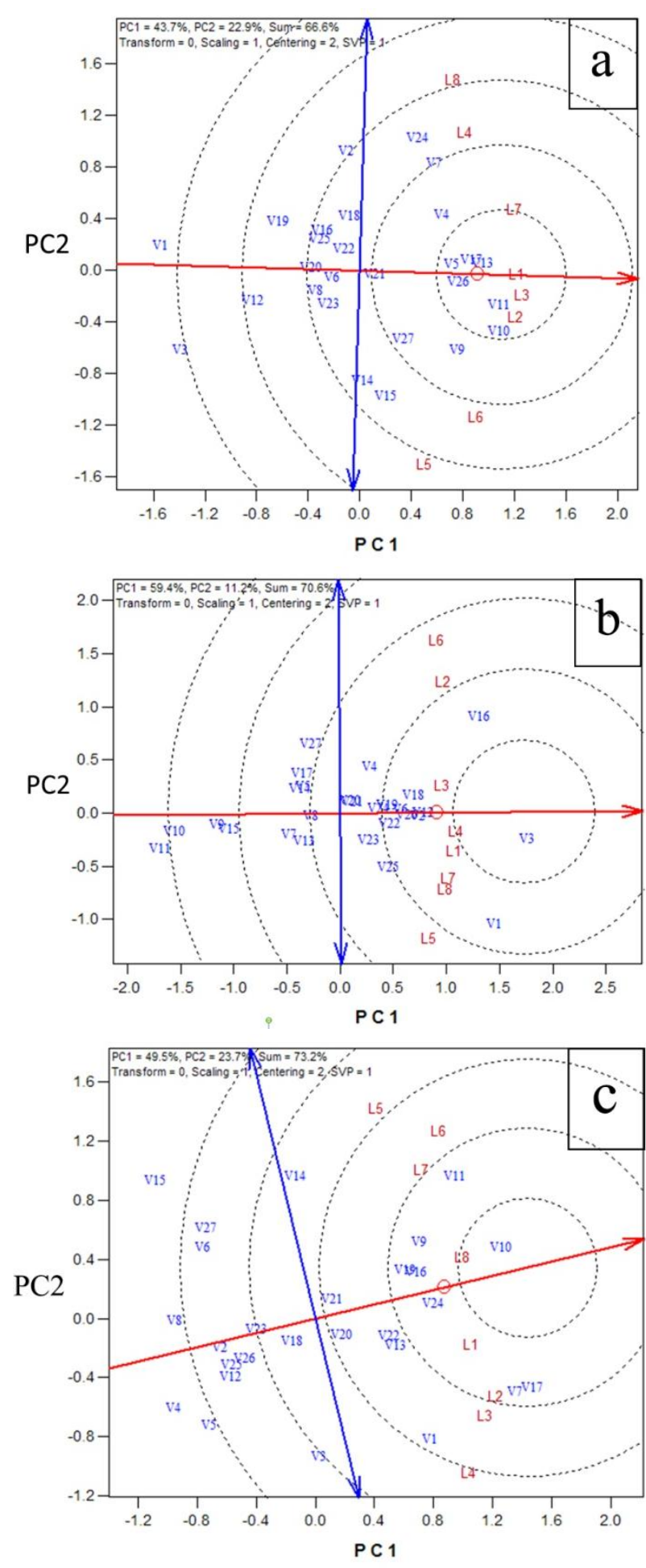

Figure 1. Number of panicles per hill (a), number of filled grains per panicle (b) and 1,000-grain weight (c) comparisons of 27 tested genotypes (see Table 1 and 2 for genotype and location descriptions, respectively) in 8 locations, using GGE-biplot (PC 1 and PC 2 are first and second principal components, respectively). 

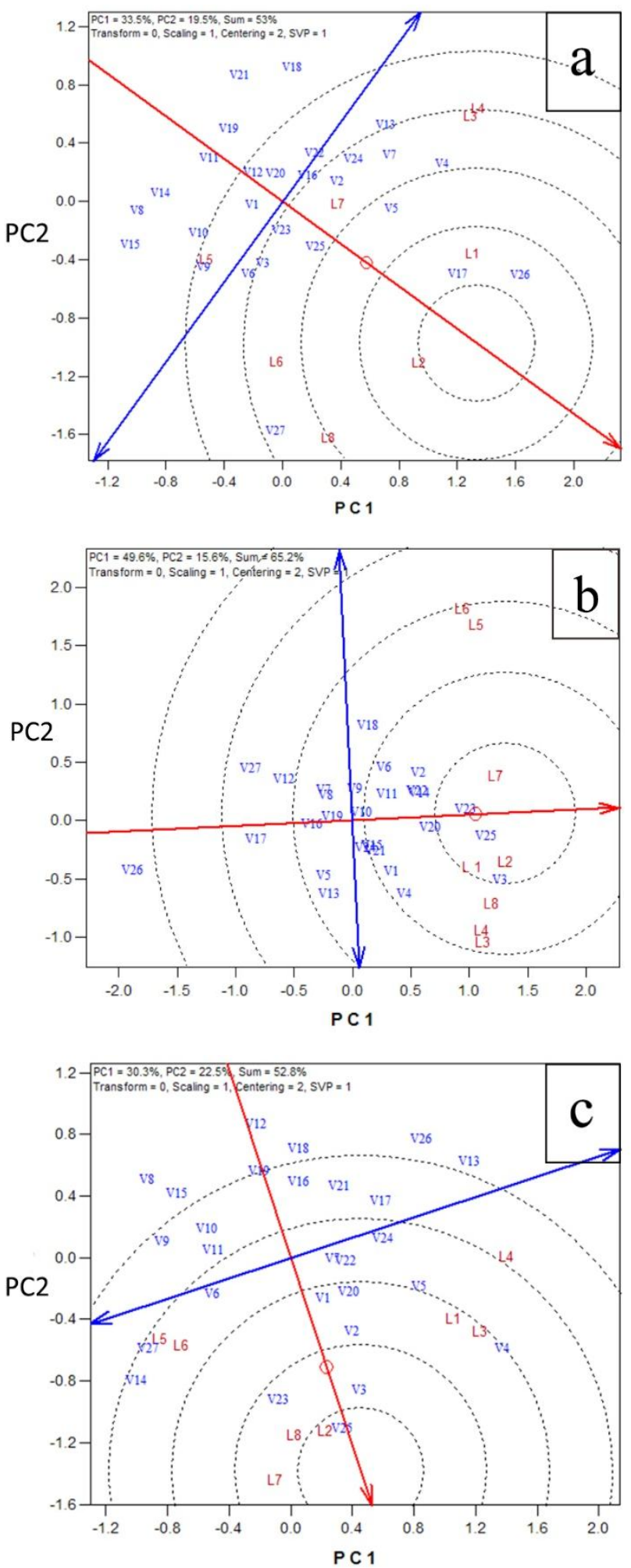

Figure 2. Yield (a), gamma oryzanol per grain weight (b) and gamma oryzanol yield (c) comparisons of 27 tested genotypes (see Table 1 and 2 for genotype and location descriptions, respectively) in 8 locations, using GGE-biplot (PC 1 and PC 2 are first and second principal components, respectively).
Although all recorded crop traits are very important for genotype evaluation across various environments, in this study, however, we paid more emphasis in grain yield, gamma oryzanol per grain weight and gamma oryzanol yield. When these three crop traits were considered, KKUGL-BL-11-001 (V25) was identified as one of desirable genotypes in term of both high mean performance and stability. The analysis results indicated that this genotype had higher general adaptability when compared to the other tested genotypes. Therefore, it would be recommended as a parental genotype for further improvement in breeding programs and for growing in a commercial scale under both lowland and upland in tropical environments.

In addition, based on the purpose of gamma oryzanol extraction for pharmaceutical and cosmetic industries, the rice genotypes with high gamma oryzanol content per grain weight are more preferable than the genotypes with high gamma oryzanol yield per area. Not only KKU-GL-BL-11001 (V25), therefore, Niewdam Gs.no. 21427 (V3) and KKU-GL-BL-06-043 (V23) would also be selected for this particular aspect. However, the results showed that these three genotypes are not the highest yielding genotypes. This indicates that breeding to improve both yield and gamma oryzanol content in rice is also the challenge for plant breeders.

\section{CONCLUSION}

The combined analysis of variance revealed that there were the significant effect of $L, G$ and $G \times L$ interaction for number of panicles per hill, number of filled grains per panicle, 1000-grain weight, grain yield, gamma oryzanol per grain weight and gamma oryzanol yield. The $\mathrm{G} \times \mathrm{L}$ interactions shared the largest portion of total variations for grain yield $(36.0 \%)$, gamma oryzanol per grain weight $(32.7 \%)$ and gamma oryzanol yield $(35.6 \%)$. The results from GGE biplot analysis indicated that KKU-GL-BL-06023 (V17), Khaokam Gs.no. 88084 (V5) and KKU-GLBL-11-001 (V25) were the superior genotypes for both high mean performance and stability in grain yield. The best genotypes for gamma oryzanol per grain weight were KKU-GL-BL-11-001 (V25) and KKU-GL-BL-06-043 (V23), whereas the desirable genotypes for gamma oryzanol yield were KKU-GL-BL-11-001 (V25), Niewdam Gs.no. 21427 (V3) and KKU-GL-BL-06-043 (V23). This would be valuable information for black glutinous rice production with high level of gamma oryzanol content and grain yield. It is also helpful information for plant breeders to improve gamma oryzanol content in rice.

\section{ACKNOWLEDGEMENTS}

This study was supported by Khon Kaen University, Thailand. Assistance in conducting the work was also received from the Plant Breeding Research Center for Sustainable Agriculture, Khon Kaen University. Acknowledgement is extended to the Thailand Research Fund (Project code : IRG5780003) and Faculty of Agriculture, Khon Kaen University for providing financial support for manuscript preparation activities. 


\section{LITERATURE CITED}

Bergman, C.J. and Z. Xu. 2003. Genotype and environment effects on tocopherol, tocotrienol, and $\gamma$-oryzanol contents of southern U.S. rice. Cereal Chem. 80(4): 446-449.

Boonsit, P., P. Pongpiachan, S. Julsrigival and D. Karladee. 2010. Gamma oryzanol content in glutinous purple rice landrace varieties. C.M.U. J. Nat. Sci. 9(1): 151-157.

Cicero, A.F.G. and A. Gaddi. 2001. Rice bran oil and $\gamma$-oryzanol in the treatment of hyperlipoproteinemias and other conditions. Phytother. Res. 15: 277-289.

Comstock, R.E. and R.H. Moll. 1963. Genotype x Environment Interactions. Symposium on Statistical Genetics and Plant Breeding. In: Statistical Genetics and Plant Breeding, ed. Hanson, W.D. and Robinson H.F., 164-196, National Academy Science National Research Council No. 982, Washington, D.C.

Freed, R., S.P. Eisensmith, S. Goetz, D. Reicosky, V.W. Smail and P. Wolberg. 1989. User's Guide to MSTAT-C. East Lansing, MI: Michigan State University, USA.

Gomez, K.A. and A.A. Gomez. 1984. Statistical Procedures for Agricultural Research. 2nd ed. John Wiley and Sons, New York. $680 \mathrm{p}$.

Juliano, C., M. Cossu, M.C. Alamanni and L. Piu. 2005. Antioxidant activity of gamma-oryzanol: Mechanism of action and its effect on oxidative stability of pharmaceutical oils. Int. J. Pharm. 299: 146-154.

Pitija, K., M. Nakornriab, T. Sriseadka, A. Vanavichit and S. Wongpornchai. 2013. Anthocyanin content and antioxidant capacity in bran extracts of some Thai black rice varieties. Int. J. Food Sci Tech. 48: 300-308.

Romagasa, I. and P.N. Fox. 1993. Genotype x environment interaction and adaptation. In: Plant breeding, Principles and prospects, ed. Hayward, M.D., Bosemark, N.O. and Romagosa I., 373-387, Chapman \& Hall, London.
Ryu, S.N., S.Z. Park and C.T. Ho. 1998. High performance liquid chromatographic determination of anthocyanin pigments in some varieties of black rice. J. Food Drug Anal. 6: 729-736.

Saenjum, C., C. Chaiyasut, S. Chansakaow, M. Suttajit and B. Sirithunyalug. 2012. Antioxidant and anti-inflammatory activities of gamma-oryzanol rich extracts from Thai purple rice bran. J. Med. Plants Res. 6(6): 1070-1077.

Sayar M.S., A.E. Anlarsal and M. Basbag. 2013. Genotypeenvironment interactions and stability analysis for dry-matter yield and seed yield in Hungarian vetch (Vicia pannonica Crantz.). Turk. J. Field Crops 18(2): 238-246.

Somsana, P., P. Wattana, B. Suriharn and J. Sanitchon. 2013. Stability and genotype by environment interactions for grain anthocyanin content of Thai black glutinous upland rice (Oryza sativa). SABRAO J. Breed. Genet. 45 (3): 523-532.

Vorarat, S., C. Managit, L. Iamthanakul, W. Soparat and N. Kamkaen. 2010. Examination of antioxidant activity and development of rice bran oil and gamma-oryzanol microemulsion. J. Health Res. 24(2): 67-72.

$\mathrm{Xu}, \mathrm{Z}$. and J.S. Godber. 1999. Purification and identification of components of $\gamma$-oryzanol in rice bran oil. J. Agric. Food Chem. 47: 2724-2728.

Xu, Z., N. Hua and J.S. Godber. 2001. Antioxidant activity of tocopherol, tocotrienols, and gamma-oryzanol components from rice bran against cholesterol oxidation accelerated by 2,2'-azobis(2-methylpropionamidine) dihydrochloride. J. Agric. Food Chem. 49: 2077-2081.

Yan, W. 2001. GGE biplot: A windows application for graphical analysis of multi-environment trail data and other types of two-way data. Agron. J. 93: 1111-1118.

Yan, W. and L.A. Hunt. 2003. Biplot analysis of multienvironment trial data. In: Quantitative Genetics, Genomics, and Plant Breeding, ed. Kang, M.S., 289-303, CAB International, Wallingford, Oxon, UK. 